\title{
M2 macrophage polarization in cutaneous polyarteritis nodosa: an immunohistochemical study
}

\author{
Gabriela Marques ${ }^{1}$, Paulo Criado ${ }^{2}$, Carla Pagliari ${ }^{3}$, Ricardo Macarenco ${ }^{4}$, Maíra Saldanha ${ }^{5}$, \\ and Thamara Morita ${ }^{1}$ \\ ${ }^{1}$ Universidade de São Paulo Hospital das Clínicas \\ ${ }^{2}$ Federal University of the ABC \\ ${ }^{3}$ Universidade de São Paulo \\ ${ }^{4}$ Hospital Israelita Albert Einstein \\ ${ }^{5}$ Fiocruz Bahia
}

July 7,2020

\begin{abstract}
Background Cutaneous polyarteritis nodosa (CPAN), a cutaneous vasculitis that affects small- and medium-sized arteries, is thought to be mediated by immune complexes. However, little is known about its etiology. Macrophages are important pathogenic factors in various vascular inflammatory diseases, including certain types of vasculitis. However, their role in CPAN remains unexplored. Objectives To describe the demographic, clinical, and laboratory findings and investigate the distribution of M1 and M2 macrophages in CPAN. Methods This was a cross-sectional study involving fourteen patients with CPAN and nine control participants. Medical records were reviewed to determine demographic and clinical data, and laboratory findings. Skin biopsies obtained during disease manifestations were evaluated immunohistochemically. M1 macrophages were identified using STAT1 expression, and M2 macrophages were identified using CD163, CD206, and CMAF expression. Results CPAN was predominant in women, and the mean age of patients was 39 years. The main dermatological lesions were ulcers, subcutaneous nodules, and livedo. Increased lipoprotein(a) levels (28\%) and a positive tuberculin skin test in $40 \%$ of the tested patients were our main laboratory findings. Direct immunofluorescence was positive in $88.9 \%$ of the cases. Immunohistochemical analysis revealed an increased percentage of M2 macrophages in CPAN patients compared with that in the control group. Conclusions This is a novel study on macrophage polarization in CPAN, where the number of cells expressing CD163 and CMAF was found to be increased. M2 macrophage predominance suggests an important role for the innate immune system in the pathophysiology of CPAN and may provide insights for potential therapeutic targets.
\end{abstract}

\section{1 | INTRODUCTION}

Cutaneous polyarteritis nodosa (CPAN) or cutaneous arteritis is a rare form of single organ vasculitis that affects small- and medium-sized arteries of the dermo-hypodermic junction and subcutaneous tissue. CPAN is a chronic and benign disease. It affects patients of all ages, and a female preponderance has been reported. The incidence and prevalence of CPAN are not well-established. Despite its good prognosis, certain cases exhibit major complications and may require careful management. ${ }^{1,2}$

CPAN is potentially mediated by immune complexes, as the presence of IgM and C3 deposits in affected arterial walls is commonly found; however, its etiology is not fully understood. ${ }^{3}$ For instance, serum IgM and IgG anti-phosphatidylserine-prothrombin complex (anti-PT/PS) antibodies were found in $81 \%$ and $31 \%$ of CPAN patients, respectively. Such findings support the hypothesis that the PS/PT complex may trigger an immune response with the formation of antigen-antibody complexes. These may initiate the classical complement pathway, leading to uncontrolled inflammation in vessel walls. ${ }^{4}$ 
CPAN may have genetic origins. CPAN was found to be in the spectrum of manifestations associated with adenosine deaminase 2 (ADA2) enzyme deficiency. ADA2 plays an important role in maintaining vascular integrity. Furthermore, ADA2 serves as a growth factor for endothelial and hematopoietic cells and acts as a promoter of monocyte proliferation and M2 macrophage polarization. ${ }^{5,6}$

The role of macrophages in vascular inflammatory diseases may provide insights for potential therapeutic targets. ${ }^{7}$ Recent studies revealed that these cells are an important pathogenic factor in the development of various types of vasculitis, including giant cell arteritis, Kawasaki disease, Henoch-Schönlein purpura, and ANCA-associated vasculitis. ${ }^{8-11}$

However, the role of macrophages in patients with CPAN has never been reported. Therefore, the objectives of this study were to investigate the distribution of M1 and M2 macrophage phenotypes in CPAN and to describe the clinical characteristics of affected patients.

\section{2 | PATIENTS AND METHODS}

\section{Patient characteristics and tissue collection}

Fourteen patients with CPAN were admitted to the Vasculitis Outpatient Clinic, Dermatology Department of University of São Paulo Medical School (São Paulo, Brazil) between January 2004 and December 2018. These patients were retrospectively analyzed in this study. Cases were selected according to the following criteria: (1) patients with a clinical and histological diagnosis of CPAN, after exclusion of internal organ involvement and (2) availability of adequate skin biopsy samples obtained from lesions located in the lower extremities during disease activity with no active treatment.

Tissue specimens were fixed in $10 \%$ formalin, step-sectioned, and stained with hematoxylin and eosin (HE). All histopathological exams were reviewed by an experienced pathologist, and the diagnosis of CPAN was made by the presence of necrotizing vasculitis of medium-sized arteries. In certain cases, Weigert ResorcinFuchsin staining for elastic fibers (WRF) was performed (Figures 1 and 2) to confirm whether the compromised vessels were arteries. Medical records were reviewed to determine demographic and clinical data and laboratory findings.

Additionally, we selected nine skin biopsy specimens as a control group. The biopsies had been previously obtained from the lower limbs of nine healthy volunteers, and were stored in the pathology laboratory of the Hospital. The control participants had a mean age of 55.4 years [standard deviation (SD) 13.5 years] and included five female participants $(55.6 \%)$ and four male participants (44.4\%). Patients and controls matched on gender, but not in age.

This study was approved by the Ethics Committee of Hospital das Clinicas of the University of São Paulo Medical School under the reference number 1.904.121, and written informed consent was obtained from all the participants.

\section{Immunohistochemical analyses}

Samples were processed for immunohistochemical evaluation. Histological sections obtained from paraffin blocks of the skin biopsy fragments were used to identify M1 and M2 macrophages in the vascular walls. To identify M1 macrophages, anti-STAT1 (SC135648, 1:50 dilution, Santa Cruz, Dallas, TX, USA) was used. To assess M2 macrophages, anti-CD163 (NCL-L-CD163, 1:400 dilution, Novocastra, Newcastle, UK), antiCD206 (15-2, 1:200 dilution, Genetex, Irvine, CA, USA), and anti-CMAF (SC7866, 1:100 dilution, Santa Cruz) were used. The working system used was labeled streptavidin biotin-horseradish peroxidase LSAB2 System-HRP (Code K0672, Dako Cytomation, Carpinteria, CA, USA), and the final product of the reaction was visualized using diaminobenzidine (Dako Cytomation). All reactions included an appropriate positive control. Negative controls were obtained by replacing the primary antibody with an IgG isotype.

The formalin-fixed, paraffin-embedded tissue samples were sliced into $4-\mu \mathrm{m}$ sections. Slides were pre-coated with $2 \% 3$-aminopropyltriethoxysilane (code A3648, Sigma, St. Louis, MO, USA). The slides were treated with xylene for wax removal and then rehydrated in a graded ethanol series $(100 \%, 95 \%$, and $70 \%$ ). To 
block endogenous peroxidase activity. The sections were next immersed in $3 \%$ hydrogen peroxide for 30 min and washed in distilled water. Antigen retrieval was performed using Target Retrieval Solution pH 9.0 (code S2367, Dako Cytomation) in a preheated $\left(95^{\circ} \mathrm{C}\right)$ water bath for $20 \mathrm{~min}$. Sections were washed in distilled water and placed in phosphate-buffered saline (PBS; pH 7.4). The slides were incubated in Molicoß skim milk (Nestlé, São Paulo, SP, Brazil) for 30 min to block nonspecific binding sites. Then, the tissue sections were incubated with primary antibodies diluted in PBS containing $1 \%$ bovine serum albumin overnight at $4^{\circ} \mathrm{C}$. The following day, tissue samples were washed with PBS pH 7.4 for $5 \mathrm{~min}$, treated with secondary antibody (Novolink Max Polymer Detection System, Leica Microsystems, Newcastle, UK) for 30 min at 22-24oC, and then assessed using streptavidin-biotin-horseradish peroxidase (LSAB-Dako). Reactions were visualized by treating the stained tissue sample with 3,3"'-diaminobenzidine-tetrahydrochloride (code D5637, SIGMA Chemical Co., St Louis, MO, USA) and $0.03 \%$ hydrogen peroxide.

Images of the sections were captured using a camera (Zeiss, AxioCam MRc, Germany) attached to a microscope (Zeiss, Axiophot, Germany). Image Pro Plus version 4.1 software (Netics Midia Cibernetico, Silver Spring, MD, EUA) was used for analysis. Cells were counted in a minimum of 15 randomly selected fields next to the affected vessels under 40x magnification. The proportion of M1 and M2 macrophages in the biopsies from CPAN patients and control participants was assessed relative to the total cell count.

\section{Statistical analysis}

Statistical analyses were performed using PASW Statistics V18 software, and graphs were generated using Microsoft Excel(r). Continuous variables are presented as the mean, median, and SD, and categorical variables are presented as absolute (n) and relative (\%) frequencies. Comparisons of continuous data between the CPAN and control groups were performed using the Chi-squared test or the Mann-Whitney U test, as applicable. $\mathrm{p}<0.05$ was considered to indicate a statistically significant difference.

\section{3 | RESULTS}

\section{Demographic and clinical findings}

The demographic, clinical, and serological findings of the patients with CPAN are summarized in Table 1. The age of the patients in the CPAN group ranged between 15 and 61 years, and the follow-up time ranged from 3 months to 14 years. There were no other diseases in $64 \%$ of the cases. Five cases had comorbidities, such as diabetes, hypothyroidism, dyslipidemia, and essential hypertension, which were not associated with CPAN. One patient was diagnosed with ductal breast carcinoma 3 years after CPAN diagnosis.

The most frequent symptoms were always present at the site of the cutaneous lesions. Patients who experienced paresthesia (burning sensation or tingling) underwent electroneuromyography, and $>40 \%$ of the cases were diagnosed with mononeuritis multiplex.

\section{Serological findings}

Full blood count, liver and renal function, serum complement concentrations, and serum protein electrophoresis pattern were normal in all patients. The cases (35\%) positive for antinuclear antibodies (ANA) exhibited a nuclear speckled pattern. All patients underwent coagulation analysis and $28 \%$ exhibited elevated lipoprotein(a) levels $(>30 \mathrm{mg} / \mathrm{dl})$.

\section{Presence of infectious agents}

Increased antistreptolysin O (ASO) titers were observed in one patient. Serology for hepatitis B virus (HBV), hepatitis $\mathrm{C}$ virus (HCV), and human immunodeficiency virus (HIV) was negative in all patients. The purified protein derivative (PPD) test was performed in 10 patients, and four patients tested positive ([?] $5 \mathrm{~mm}$ ). These patients were subsequently referred to the infectious diseases clinic, and treatment for tuberculosis was initiated with isoniazid, rifampicin, ethambutol, and pyrazinamide.

\section{Immunofluorescence study}


Direct immunofluorescence, previously performed on nine of the CPAN skin biopsy samples, was positive in $89 \%$ of the cases. The most frequent deposits were $\operatorname{IgM}(66.7 \%)$ and $\mathrm{C} 3(66.7 \%)$, followed by $\operatorname{IgA}(22.2 \%)$ and $\operatorname{IgG}(11.1 \%)$.

\section{Macrophages in skin biopsies}

Macrophage markers associated with M1 cells were not found in either group. M2 markers were present in the CPAN group. As shown in Table 2 and Figure 2, the frequency of cells expressing CD163 and CMAF markers was significantly higher in patients with CPAN than in the controls. Representative images of the immunohistochemical staining are presented in Figure 4.

\section{4 | DISCUSSION}

We presented an evaluation of the demographic, clinical, and laboratory findings of 14 patients with CPAN seen in our clinic over a fifteen-year period. We also investigated the potential role of innate immunity in the disease by evaluating macrophage polarization in skin biopsies.

Unlike systemic PN, the cutaneous variant more frequently develops in women, with some studies showing a prevalence of up to $90 \%$. The female to male (F/M) ratio ranges from $1.6-13 ;^{1,12,13}$ in the present study, a 3.6:1 F/M ratio was observed. Although the disease affects patients of all ages, young adults tend to be the most affected, ${ }^{1,12,14}$ as was observed in our results. The median age of onset can range from 35 to 45 years old.

The skin manifestations observed in the patients in the present study were similar to those reported in the literature ${ }^{4,12,15,16}$ however, the frequencies were somewhat different. The prevalence of ulcers was higher, while that of subcutaneous nodules was lower in this study. Considering that ulcers usually develop in nodules, and access to public health services is limited in Brazil, we believe that diagnosis in a more advanced phase of the disease partly influenced these results. ${ }^{15,17,18}$

Livedo can occur in $40-80 \%$ of CPAN cases and can sometimes be the first manifestation of the disease; ${ }^{13,17}$ our findings corroborate these figures. While some authors use the expressions livedo reticular and livedo racemosa interchangeably, others consider the former as a physiological manifestation that is secondary to vasoconstriction, while the latter condition presents as an irregular pattern of skin discoloration associated with pathological processes such thrombosis and vasculitis. ${ }^{16,19}$

As shown in this study, atrophie blanche may occur in some cases of CPAN. This type of scar is not pathognomonic of livedoid vasculopathy and occurs in disorders such as venous stasis, antiphospholipid antibody syndrome, and cutaneous vasculitis. The histological findings of atrophie blanche, characterized by a segmental hyaline degeneration of blood vessel walls with an intravascular deposition of fibrin, are usually present in the dermis. To exclude CPAN in patients with atrophie blanche, repeated and deep biopsies are often required to obtain samples of medium-sized arteries. ${ }^{15,20}$

As observed in other cases of CPAN, the legs were affected in all the patients. Although ulcers and nodules could be found in the upper limbs and trunk, in our cases, these sites were affected only by livedo racemose in only three patients. Previous studies suggest that patients with CPAN may have a local circulation dysfunction, accounting for the predominance of skin manifestations in the lower extremities. ${ }^{12,13,15}$

The prevalence of mononeuritis multiplex confirmed by electroneuromyography in our patients (21\%) is similar to those in other studies. ${ }^{13,16,17}$ An ischemic mechanism, secondary to the presence of necrotizing vasculitis of vasa nervorum, may be the main cause of mononeuritis multiplex in CPAN. ${ }^{21,22}$ Nevertheless, it is important to consider that confirmation through electroneuromyography in our study was performed only in patients with peripheral neuropathy symptoms. This limitation may suggest that the prevalence of mononeuritis multiplex could be even higher in CPAN if routine electrophysiological studies were to be performed. Another finding was that among the three patients with mononeuritis, two were males. This result requires further investigation in larger studies, preferably with a prospective design, in order to verify if mononeuritis is more prevalent in men with CPAN. 
The absence of extracutaneous symptoms such as fever, arthralgia, and weight loss contradicts the literature, with studies describing extracutaneous manifestations in up to $64 \%$ of patients with CPAN. As a limitation of a retrospective review, we believe that extracutaneous symptoms may not have been adequately registered in medical records and, therefore, discrepancy between our data and that in the literature may have been unintentionally created.

General laboratory results show only a few abnormalities in patients with CPAN. The increased levels of lipoprotein(a) in $28 \%$ of our patients with CPAN is a novel observation that is significantly associated with the disease. The plasma lipoprotein(a) level is considered a potential atherogenic and thrombogenic risk factor. Alterations in plasma lipoprotein(a) level have been associated with the pathogenesis of vasculopathies such as livedoid vasculopathy and vasculitis associated with Behcet's disease. ${ }^{23-25}$ Studies with a larger number of patients are required to establish whether lipoprotein(a) has a thrombotic role in the pathogenesis of CPAN.

Contrary to what is observed in PN, HBV and HCV infections were not associated with CPAN in our study. The increased ASO titers in a 17-year-old patient is in line with other studies and may have been attributed to streptococcal infection, which may occur in young patients with CPAN. The association with Mycobacterium tuberculosis has been reported in a few cases of CPAN. ${ }^{13,26-28}$ Although tuberculosis is endemic in Brazil, the positive PPD test in $28 \%$ of patients with CPAN patients is a relevant finding and should be further investigated.

Since CPAN has a prolonged course, with variable periods of relapse and remission, a follow-up is always recommended. None of our cases progressed to PN after a mean follow-up of $\sim 7$ years, which supports the notion that the cutaneous variant is distinct from PN. However, as there are reported cases of disease progression to the systemic form after 18 years, a longer follow-up is necessary. ${ }^{12,17,18,29}$

A comparative summary of the epidemiological and clinical data observed in the main published works that evaluated cases of CPAN is shown in Table 3.

Macrophages in tissue samples can be identified by the investigation of transcription factors or macrophagespecific proteins such as enzymes, transmembrane proteins, scavenger receptors, or cytokines. ${ }^{30,31}$ Although there are distinct markers that may be used to distinguish between M1 and M2 macrophages, STAT1, CD163, CD206, and CMAF were investigated in this study as these are well-established in the literature and have been used to investigate macrophage polarization in several diseases. ${ }^{32,33}$

M1 macrophages are usually implicated in autoimmune inflammatory diseases. Therefore, it would be plausible to verify the same trend in CPAN. Surprisingly, our data revealed that M2 macrophages were more frequent than M1 macrophages in the skin lesions of patients with CPAN. This finding may be justified by the fact that many patients could have been treated with at least one medication prior to referral to our clinic. During the first medical consultation, certain patients were unable to accurately provide their drug history. It is not possible to completely rule out the prior use of oral corticosteroids, which could influence the activation of M2 macrophages. However, Some authors advocate that the use of corticosteroids would have little to no influence on the activation of M2 macrophages. ${ }^{11}$

The presence of M2 macrophages has been previously reported in other autoimmune inflammatory diseases, and in different types of vasculitis (Table 4). Furthermore, previous studies suggest that these cells may predict patient outcomes. In Henoch-Schonlein purpura nephritis, the number of M2 macrophages was related to elevated serum creatinine, chronic renal failure, and crescent formation. A similar trend was observed in patients with ANCA-associated pauci-immune glomerulonephritis, suggesting that M2 cells may be indicative of a poor prognosis in vasculitis with renal impairment. ${ }^{9,34}$

The hypothesis that M2 macrophages could influence the interval and frequency of relapses in chronic vasculitis has also been suggested. Ohlsson et al compared the number of M2 cells in patients diagnosed with microscopic polyangiitis (MPA) and granulomatosis with polyangiitis (GPA) during the active and remission phases. They found a significant difference in the concentration of M2 macrophages between active cases of MPA and those in remission. However, this trend was not observed in patients with GPA, 
and M2 polarization was observed during remission in these patients. Therefore, there is an undefined role of M2 cells in periods of relapse in certain types of vasculitis. ${ }^{11}$

The predominance of M2 macrophages suggests a possible role of the innate immune system in CPAN and may also provide insights for potential therapeutic targets. Future studies with larger samples are required to establish a more accurate role of macrophages in vascular inflammation associated with CPAN.

The limitations of our study are mainly associated with its cross-sectional nature. Longitudinal evaluation of the participants with skin biopsy samples collected during disease activity and after remission could better explain the role of M2 cells in the evolution of CPAN. Furthermore, due to the difficulty in obtaining skin biopsies from healthy controls, it was not possible to age-match the groups.

\section{5 | REFERENCES}

1 Furukawa F. Cutaneous polyarteritis nodosa: an update. Ann Vasc Dis 2012; 5 :282-8.

2 Morgan AJ, Schwartz RA. Cutaneous polyarteritis nodosa: a comprehensive review. Int J Dermatol 2010; $49: 750-6$.

3 Diaz-Perez JL, Schroeter AL, Winkelmann RK. Cutaneous periarteritis nodosa: immunofluorescence studies. Arch Dermatol 1980;116:56-8.

4 Kawakami T, Yamazaki M, Mizoguchi M, Soma Y. High titer of anti-phosphatidylserine-prothrombin complex antibodies in patients with cutaneous polyarteritis nodosa. Arthritis Rheum 2007;57:1507-13.

5 Gonzalez Santiago TM, Zavialov A, Saarela J, et al.Dermatologic Features of ADA2 Deficiency in Cutaneous Polyarteritis Nodosa. JAMA Dermatol 2015; 151 :1230-4.

6 Caorsi R, Penco F, Grossi A, et al. ADA2 deficiency (DADA2) as an unrecognised cause of early onset polyarteritis nodosa and stroke: a multicentre national study. Ann Rheum Dis 2017;76 :1648-56.

7 Shirai T, Hilhorst M, Harrison DG, et al. Macrophages in vascular inflammation-From atherosclerosis to vasculitis.Autoimmunity $2015 ; 48: 139-51$.

8 Ciccia F, Alessandro R, Rizzo A, et al. IL-33 is overexpressed in the inflamed arteries of patients with giant cell arteritis.Ann Rheum Dis 2013; 72 :258-64.

9 Kim J, Choi S-E, Lee KH, et al. Tubulointerstitial Infiltration of M2 Macrophages in Henoch-Schonlein Purpura Nephritis Indicates the Presence of Glomerular Crescents and Bad Clinical Parameters.BioMed Res Int 2019; 2019 . doi:10.1155/2019/8579619.

10 Ohashi R, Fukazawa R, Shimizu A, et al. M1 macrophage is the predominant phenotype in coronary artery lesions following Kawasaki disease. Vasc Med Lond Engl 2019; 24 :484-92.

11 Ohlsson SM, Linge CP, Gullstrand B, et al. Serum from patients with systemic vasculitis induces alternatively activated macrophage M2c polarization. Clin Immunol Orlando Fla 2014; 152 :10-9.

12 Ikeda T, Kawakami T, Arimura Y, et al. Survey of Japanese dermatological vasculitis specialists on cases of cutaneous arteritis (cutaneous polyarteritis nodosa). J Dermatol 2020. doi:10.1111/1346-8138.15273.

13 Ishiguro N, Kawashima M. Cutaneous polyarteritis nodosa: a report of 16 cases with clinical and histopathological analysis and a review of the published work. J Dermatol 2010; 37 :85-93.

14 Criado PR, Marques GF, Morita TCAB, de Carvalho JF. Epidemiological, clinical and laboratory profiles of cutaneous polyarteritis nodosa patients: Report of 22 cases and literature review. Autoimmun Rev2016; $15: 558-63$.

15 Morimoto A, Chen K-R. Reappraisal of histopathology of cutaneous polyarteritis nodosa. J Cutan Pathol $2016 ; 43: 1131-8$. 
16 Buffiere-Morgado A, Battistella M, Vignon-Pennamen M-D, et al.Relationship between cutaneous polyarteritis nodosa (cPAN) and macular lymphocytic arteritis (MLA): Blinded histologic assessment of 35 cPAN cases. J Am Acad Dermatol 2015; 73 :1013-20.

17 Kawakami T, Okudaira A, Okano T, et al. Treatment for cutaneous arteritis patients with mononeuritis multiplex and elevated C-reactive protein. J Dermatol 2013; 40 :955-61.

18 Daoud MS, Hutton KP, Gibson LE. Cutaneous periarteritis nodosa: a clinicopathological study of 79 cases. Br J Dermatol 1997;136:706-13.

19 Pincelli MS, Echavarria AMJ, Criado PR, et al. Livedo Racemosa: Clinical, Laboratory, and Histopathological Findings in 33 Patients. Int J Low Extrem Wounds 2020; :1534734619896938.

20 Mimouni D, Ng PP, Rencic A, et al. Cutaneous polyarteritis nodosa in patients presenting with atrophie blanche. Br J Dermatol 2003; 148 :789-94.

21 Said G, Lacroix-Ciaudo C, Fujimura H, et al. The peripheral neuropathy of necrotizing arteritis: a clinicopathological study. Ann Neurol 1988; 23 :461-5.

22 James J, Jose J, Thulaseedharan NK. Acute Necrotizing Vasculitic Neuropathy due to Polyarteritis Nodosa. Oman Med $J$ 2018;33 :253-5.

23 Criado PR, Espinell DPS, Barreto P, et al. Lipoprotein(a) and livedoid vasculopathy: A new thrombophilic factor? Med Hypotheses 2015; 85 :670-4.

24 Espinel DPGS, Di Giacomo TB, Pincelli TP, et al. Analysis of serum levels and cutaneous expression of lipoprotein (a) in 38 patients with livedoid vasculopathy. J Cutan Pathol 2017;44:1033-7.

25 Balik O, Gur G, Lenk N, et al. Serum lipoprotein (a) levels and Behcet's disease: is there an association? Int $J$ Dermatol2007; 46 :827-9.

26 Imanishi H, Tsuruta D, Oshimo T, et al. Cutaneous polyarteritis nodosa induced by Mycobacterium tuberculosis. J Dermatol 2012; 39 :738-9.

27 Tang MBY, Liew KVS, Ng PPL, et al. Cutaneous polyarteritis nodosa associated with precore mutant hepatitis B infection. Br J Dermatol 2003; 149 :914-5.

28 Diez-Picazo LD, Yebra M, Mellor S, Suarez E. [Hepatitis C and cutaneous polyarteritis nodosa]. Med Clin (Barc) 2002;118:278.

29 Chen KR. Cutaneous polyarteritis nodosa: a clinical and histopathological study of 20 cases. J Dermatol $1989 ; 16: 429-42$.

30 Liu Y-C, Zou X-B, Chai Y-F, Yao Y-M. Macrophage polarization in inflammatory diseases. Int J Biol Sci $2014 ; 10: 520-9$.

31 Mills CD. M1 and M2 Macrophages: Oracles of Health and Disease.Crit Rev Immunol 2012; 32 :463-88.

32 Barros MHM, Hauck F, Dreyer JH, et al. Macrophage polarisation: an immunohistochemical approach for identifying M1 and M2 macrophages. PloS One 2013; 8 :e80908.

33 Bhattacharya S, Aggarwal A. M2 macrophages and their role in rheumatic diseases. Rheumatol Int 2019; $39: 769-80$.

34 Zhao L, David MZ, Hyjek E, et al. M2 macrophage infiltrates in the early stages of ANCA-associated pauci-immune necrotizing GN.Clin J Am Soc Nephrol CJASN 2015; 10 :54-62.

35 Park J, Lee EB, Song YW. Decreased tumour necrosis factor- $\alpha$ production by monocytes of granulomatosis with polyangiitis. Scand J Rheumatol 2014; 43 :403-8. 
36 de Souza AWS, van Timmeren M, Sanders J-S, et al. M2 macrophage is the predominant phenotype in airways inflammatory lesions in patients with granulomatosis with polyangiitis. Arthritis Res Ther 2017; 19 $: 100$.

37 Nakashima C, Otsuka A, Kitayama N, et al. Basophil and M2 macrophage infiltration in lesional skin of eosinophilic granulomatosis with polyangiitis. Eur J Dermatol EJD 2017; 27 :552-3.

\section{FIGURES}

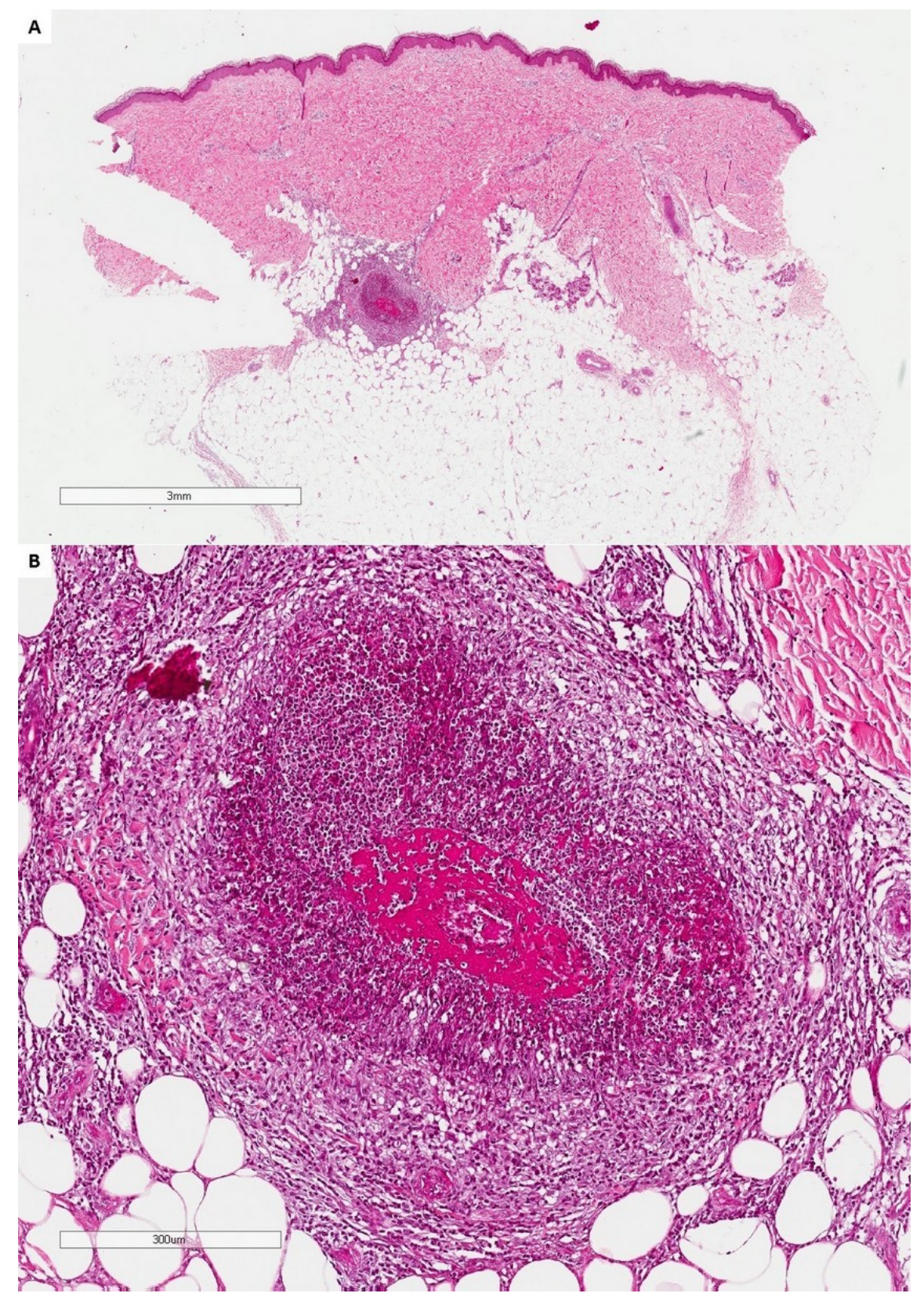

Figure 1 - (A) Necrotizing vasculitis of a muscular artery in the subcutaneous (scanning magnification, HE); (B) Details of the perivascular neutrophilic inflammatory infiltrate with fibrinoid degeneration of vessel walls and lumen occlusion (HE, 200X). 
Figure 2 - (A) Medium-sized artery in subcutaneous tissue exhibiting perivascular inflammatory infiltrate with a complete disruption of vessel wall anatomy, fibrinoid necrosis, and fibrin deposits in the vessels (HE, 100X); (B) Staining for elastic fibers showing destruction of the internal elastic lamina (WRF, 100X).
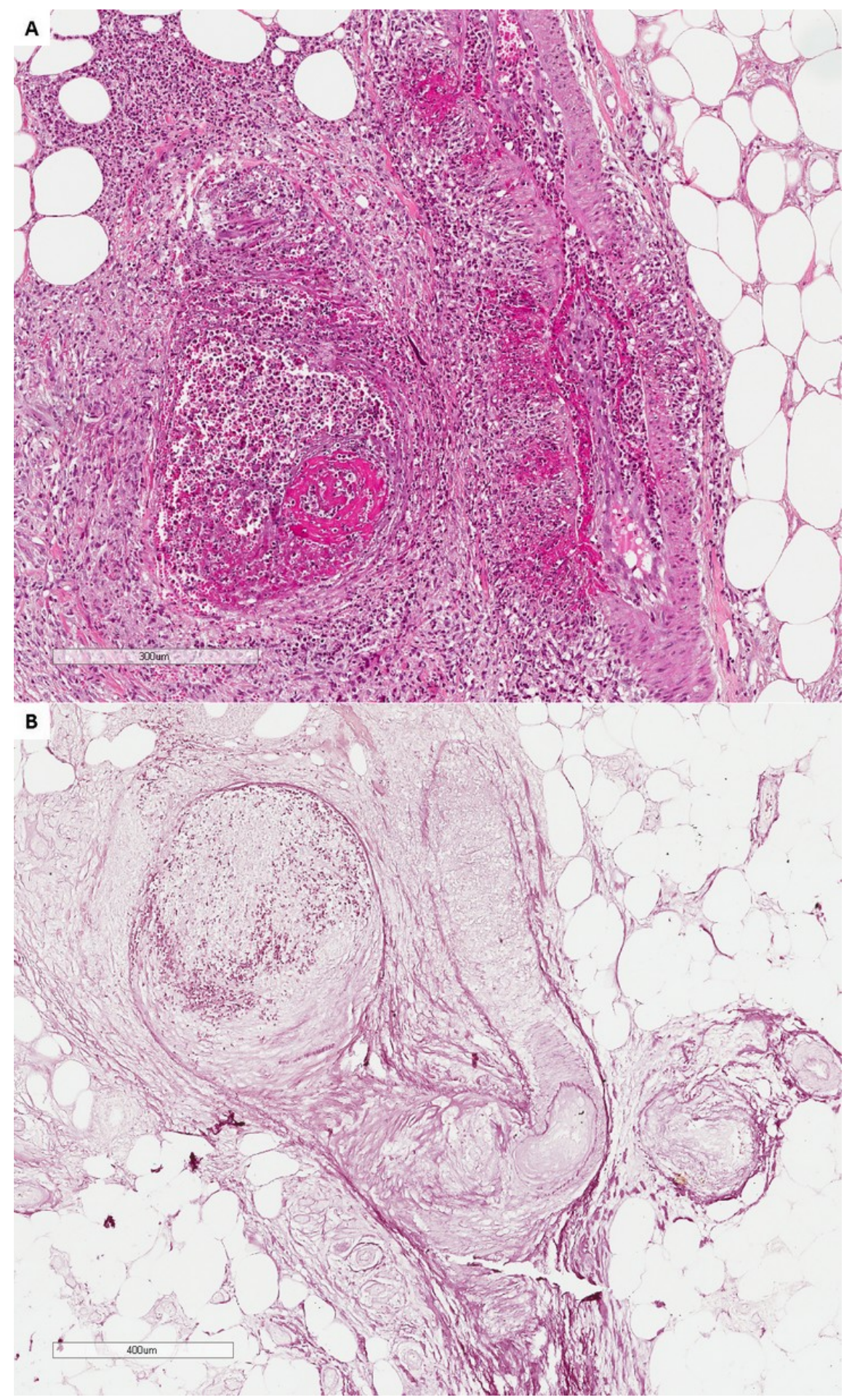
Figure 3 - Comparison of M1 and M2 macrophage scores in skin biopsies obtained from patients with cutaneous polyarteritis nodosa (CPAN) and controls.

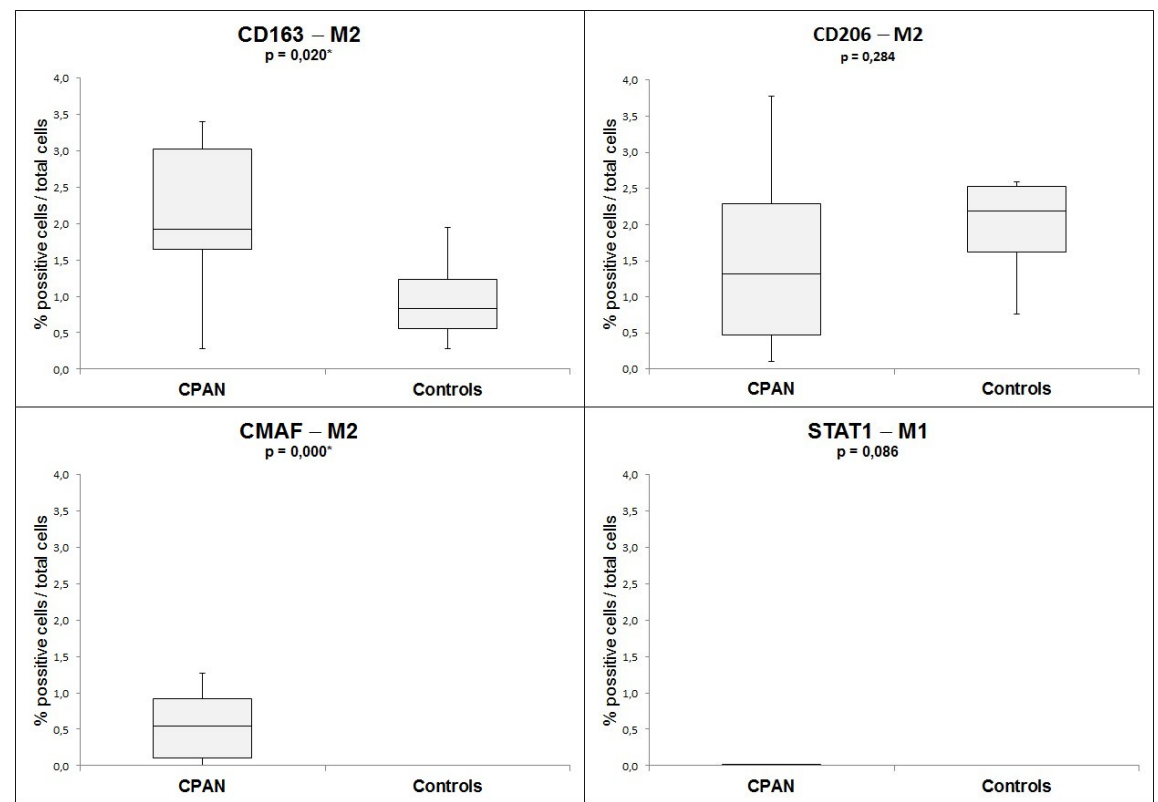

Figure 4 - Representative images of immunostaining for M1 and M2 macrophages. The expression of CD163, CD206, and CMAF is evident, while no expression of STAT1 was observed. 


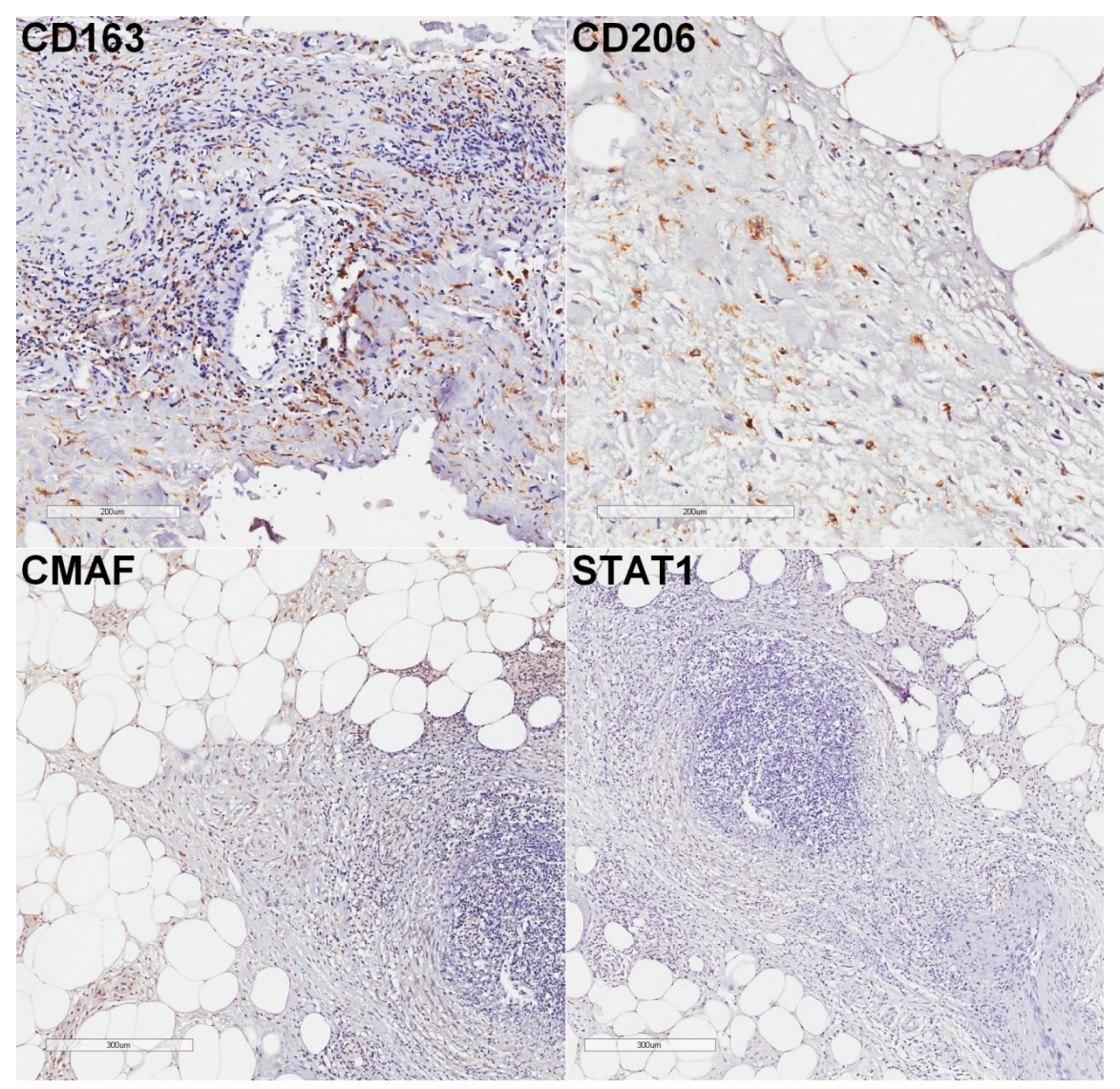

\section{TABLES}

Table 1 - Features of patients with cutaneous polyarteritis nodosa (CPAN) in the present study

\begin{tabular}{ll}
\hline Variable & Results $(\mathbf{n}=\mathbf{1 4})$ \\
\hline Female, $\mathrm{n}(\%)$ & $11(78,6 \%)$ \\
Mean age, years $\pm \mathrm{SD}$ & $39,36 \pm 14,5$ \\
Skin signs, n (\%) & $8(57,1 \%)$ \\
Ulcers & $7(50,0 \%)$ \\
Subcutaneous nodules & $6(42,9 \%)$ \\
Livedo racemosa & $5(35,7 \%)$ \\
Subcutaneous nodules & $3(21,4 \%)$ \\
Atrophie blanche & $8(57,1 \%)$ \\
Purpura & \\
Symptoms in skin lesions, n $(\%)$ & $3(21,4 \%)$ \\
None & $11(78,6 \%)$ \\
Pain & $7(50,0 \%)$ \\
Paresthesia & \\
Target skin sites, n (\%) & $14(100 \%)$ \\
Lower extremities & $0(0 \%)$ \\
Extracutaneous symptoms, n $(\%)$ & $3(21,4 \%)$ \\
Mononeuritis multiplex, $\mathrm{n}(\%)$ & \\
Serological findings, $\mathrm{n}(\%)$ &
\end{tabular}




\begin{tabular}{ll}
\hline Variable & Results $(\mathbf{n} \mathbf{- 1 4})$ \\
\hline Complement Consumption & $0(0 \%)$ \\
ANA test positivity & $5(35,7 \%)$ \\
ANCA positivity (IFI) & $0(0 \%)$ \\
Coagulation analysis (\%) & $4(28,6 \%)$ \\
Elevated lipoprotein(a) levels & $2(14,3 \%)$ \\
Increased D-dimer levels & $1(7,1 \%)$ \\
Positive anti-cardiolipin antibody & $1(7,1 \%)$ \\
Increased fibrinogen & $1(7,1 \%)$ \\
Infectious agents (\%) & $0(0 \%)$ \\
Streptococcus & $0(0 \%)$ \\
HBV & $0(0 \%)$ \\
HCV & $4(28,6 \%)$ \\
HIV & $82,57 \pm 42,8$ \\
Mycobacterium tuberculosis & $0(0 \%)$ \\
Mean follow-up, months \pm SD & \\
Progression to PAN &
\end{tabular}

ANA, Antinuclear Antibodies; ANCA, Antineutrophil cytoplasmic antibody; HBV, hepatitis B virus; HCV, hepatitis C virus; HIV, human immunodeficiency virus; IFI, indirect immunofluorescence; PNA, polyarteritis nodosa; PPD, purified protein derivative.

Table 2 - Evaluation of macrophage scores in skin biopsies from CPAN and control groups

\begin{tabular}{lllllll}
\hline & CPAN $(\mathbf{n}=\mathbf{1 4})$ & CPAN $(\mathbf{n}=\mathbf{1 4})$ & CPAN $(\mathbf{n}=\mathbf{1 4})$ & Controls $(\mathbf{n}=\mathbf{9})$ & Controls $(\mathbf{n}=\mathbf{9})$ & $\mathbf{C}$ \\
\hline Variable & Mean & Median & SD & Mean & Median & S \\
CD163, \% & 2,466600 & 1,925350 & 2,0418141 & 1,049644 & 0,834200 & 0, \\
CD206, \% & 1,522679 & 1,314000 & 1,2466861 & 1,916822 & 2,190000 & 0, \\
CMAF, \% & 0,629729 & 0,546500 & 0,6960948 & 0,000000 & 0,000000 & 0, \\
STAT1, \% & 0,012650 & 0,000000 & 0,0330935 & 0,000000 & 0,000000 & 0, \\
\hline
\end{tabular}

CPAN, cutaneous polyarteritis nodosa; SD, standard deviation. * Significant differences.

Table 3 - Main epidemiological and clinical data of patients with CPAN published in the literature and in the present case series

\begin{tabular}{|c|c|c|c|c|c|c|c|}
\hline $\begin{array}{l}\text { Author } \\
\text { (year) } \\
\text { No cases }\end{array}$ & $\begin{array}{l}\text { Ikeda et } \\
\text { al. } \\
(2020)^{12} \\
\mathbf{n}=84\end{array}$ & $\begin{array}{l}\text { Morimoto } \\
\text { et al. } \\
(2016)^{15} \\
\mathbf{n}=21\end{array}$ & $\begin{array}{l}\text { Morgado } \\
\text { et al. } \\
(2015)^{16} \\
\mathrm{n}=35\end{array}$ & $\begin{array}{l}\text { Kawakami } \\
\text { et al. } \\
(2013)^{17} \\
n=101\end{array}$ & $\begin{array}{l}\text { Furukawa } \\
\text { et al. } \\
(2012)^{1} \mathrm{n} \\
=22\end{array}$ & $\begin{array}{l}\text { Ishiguro } \\
\text { et al. } \\
(2010)^{13} \\
\mathrm{n}=16\end{array}$ & $\begin{array}{l}\text { Marques } \\
\text { et al. } \\
(2020) \mathrm{n} \\
=14\end{array}$ \\
\hline Woman & $70 \%$ & $81 \%$ & $80 \%$ & $70 \%$ & $86 \%$ & $87 \%$ & $78 \%$ \\
\hline $\begin{array}{l}\mathrm{F} / \mathrm{M} \\
\text { ratio }\end{array}$ & $2,3: 1$ & $4: 1$ & $4: 1$ & $2,4: 1$ & $6: 1$ & $7: 1$ & $3,6: 1$ \\
\hline $\begin{array}{l}\text { Mean age } \\
\text { (years) }\end{array}$ & 45 & 44 & 45 & 45 & 48 & 36 & 39 \\
\hline Nodule & $24 \%$ & $100 \%$ & $74 \%$ & $100 \%$ & $86 \%$ & $62 \%$ & $50 \%$ \\
\hline Ulceration & $30 \%$ & $1 \%$ & $14 \%$ & $49 \%$ & $22 \%$ & $18 \%$ & $57 \%$ \\
\hline Livedo & $61 \%$ & $62 \%$ & $74 \%$ & $84 \%$ & $45 \%$ & $43 \%$ & $43 \%$ \\
\hline
\end{tabular}




\begin{tabular}{|c|c|c|c|c|c|c|c|}
\hline $\begin{array}{l}\text { Author } \\
\text { (year) } \\
\text { No cases } \\
\end{array}$ & $\begin{array}{l}\text { Ikeda et } \\
\text { al. } \\
(2020)^{12} \\
\mathrm{n}=84\end{array}$ & $\begin{array}{l}\text { Morimoto } \\
\text { et al. } \\
(2016)^{15} \\
\mathrm{n}=21\end{array}$ & $\begin{array}{l}\text { Morgado } \\
\text { et al. } \\
(2015)^{16} \\
\mathrm{n}=35\end{array}$ & $\begin{array}{l}\text { Kawakami } \\
\text { et al. } \\
(2013)^{17} \\
\mathrm{n}=101\end{array}$ & $\begin{array}{l}\text { Furukawa } \\
\text { et al. } \\
(2012)^{1} \mathrm{n} \\
=22\end{array}$ & $\begin{array}{l}\text { Ishiguro } \\
\text { et al. } \\
(2010)^{13} \\
\mathrm{n}=16\end{array}$ & $\begin{array}{l}\text { Marques } \\
\text { et al. } \\
(2020) \mathrm{n} \\
=14\end{array}$ \\
\hline $\begin{array}{l}\text { Atrophie } \\
\text { blanche }\end{array}$ & - & $14 \%$ & - & - & - & - & $36 \%$ \\
\hline Purpura & $40 \%$ & $9 \%$ & - & $67 \%$ & $45 \%$ & $25 \%$ & $21 \%$ \\
\hline $\begin{array}{l}\text { Legs } \\
\text { compro- } \\
\text { mised }\end{array}$ & $85 \%$ & $100 \%$ & $100 \%$ & $100 \%$ & $100 \%$ & $100 \%$ & $100 \%$ \\
\hline $\begin{array}{l}\text { Extracutaneoi } \\
\text { symp- } \\
\text { toms }\end{array}$ & $\mathrm{A} \%$ & $24 \%$ & $31 \%$ & $67 \%$ & $64 \%$ & $66 \%$ & $0 \%$ \\
\hline $\begin{array}{l}\text { Mononeuritis } \\
\text { multi- } \\
\text { plex }\end{array}$ & & - & $9 \%$ & $57 \%$ & - & $22 \%$ & $21 \%$ \\
\hline $\begin{array}{l}\text { Progression } \\
\text { to PN }\end{array}$ & $0 \%$ & $0 \%$ & $0 \%$ & $0 \%$ & $0 \%$ & $0 \%$ & $0 \%$ \\
\hline
\end{tabular}

F, Female; M, Male; PN, Polyarteritis nodosa; -, not available.

Table 4 - Vasculitis associated with macrophages and their phenotype

\begin{tabular}{lllll}
\hline Type of vasculitis & No cases & Sample type & Macrophage phenotype & Reference \\
\hline GCA & 20 & Temporal artery biopsy & M1 and M2 & Ciccia F et al. $(2013)^{8}$ \\
GPA & 22 & Lung biopsy & M2 & Park J et al. $(2014)^{35}$ \\
MPA & 10 & Blood & M2 & Ohlsson SM et al. $(2014)^{11}$ \\
GPA & 13 & Blood & M2 & Ohlsson SM et al. $(2014)^{11}$ \\
GPA & 35 & Respiratory tract biopsy & M2 & de Souza AWS et al. $(2017)^{36}$ \\
EGPA & 1 & Skin biopsy & M2 & Nakashima C et al. $(2017)^{37}$ \\
Henoch-Schönlein & 49 & Renal biopsy & M2 & Kim J et al. $(2019)^{9}$ \\
Kawasaki disease & 5 & Coronary artery biopsy & M1 & Ohashi R et al. $(2019)^{10}$ \\
\hline
\end{tabular}

GCA: Giant cell arteritis; GPA: Granulomatosis with polyangiitis; MPA: Microscopic polyangiitis; EGPA: Eosinophilic granulomatosis with polyangiitis. 